Research Article

\title{
Optimization of Sports Event Management System Based on Wireless Sensor Network
}

\author{
Yuan Zhang, Xuanli Zhao ${ }^{(D)}$, Jing Shen, Kaixuan Shi, and Yang Yu \\ Department of Physical Education, China University of Geosciences (Beijing), Beijing 100083, China \\ Correspondence should be addressed to Xuanli Zhao; 2012010033@cugb.edu.cn
}

Received 25 September 2021; Accepted 18 October 2021; Published 2 November 2021

Academic Editor: Guolong Shi

Copyright ( 2021 Yuan Zhang et al. This is an open access article distributed under the Creative Commons Attribution License, which permits unrestricted use, distribution, and reproduction in any medium, provided the original work is properly cited.

\begin{abstract}
In recent years, wireless sensor network technology has developed rapidly and its role in managing systems for sports events has been widely used. Wireless sensor networks not only have low wiring cost, high monitoring accuracy, and good fault tolerance but also can be monitored remotely and have outstanding advantages in fault diagnosis and safety monitoring. In this paper, firstly, the wireless sensor network hierarchical routing protocol is studied and its network model and workflow are analyzed; according to the energy consumption of the wireless sensor network, the selection method of the optimal number of cluster heads is proposed to analyze the advantages and disadvantages existing in the protocol. Secondly, the improvement of the routing protocol is proposed to address the problems of uneven distribution of cluster heads and cluster head election without considering the residual energy of nodes in the protocol. When dividing clusters, the number of neighboring nodes is considered so that cluster heads are distributed more evenly in the network; when electing cluster heads, the residual energy of nodes in the cluster is considered to balance the whole network load, and when electing cluster heads, the residual energy of nodes in the cluster is considered to balance the whole network load. Finally, simulation experiments are conducted in this paper using simulation software, and the simulation results show that the data fusion algorithm is more effective than the protocol in reducing the average energy consumption of nodes and extending the network lifetime; these features make wireless sensors more beneficial for better management of sporting events as well as better optimization.
\end{abstract}

\section{Introduction}

With the continuous development of wireless sensor network technology, smartphone communication technology, and Internet information technology, remote wireless mobile sporting event monitoring systems will become one of the mainstream intelligent sporting event networks. Smart sports event technology is the use of wireless communication technology, sensor network technology, Internet technology, embedded technology, and computer technology to connect various electrical devices at home through the network to form a set of Internet of the Things control systems. With this system, people can remotely monitor the electrical equipment at home simply by using mobile phones, providing an efficient solution for intelligent home appliance control and security and antitheft systems [1], which allows for a certain degree of optimization of sporting event management. Wireless sensor network and wireless communication technology in the form of self-group multihop monitoring of a variety of equipment and monitoring targets, while through the embedded processing technology, but also the monitoring of the data can be real-time analysis and processing, has a very broad application prospect. With the development of the times, people's quality of life continues to improve, the intelligence of intelligent sports events is also getting higher and higher, and intelligent sports events become the focus of discussion and research nowadays. At present, most of the applications related to intelligent sports events in the market are mainly connected to the network in a wired way, which is not only costly and troublesome to the wire but also when the number of node devices grows exponentially, the wired networking method may not even be possible under certain conditions.

In some remote mountainous areas in the wild, the inconvenience caused by this networking method will be more obvious. The various shortcomings of wired networking are effectively solved by the integration of wireless sensor 
networks and wireless communication technology. With the development of wireless LAN and wireless communication technology, remote wireless monitoring of sporting event systems will become the main trend of intelligent sporting event networks. Smart sporting events are the use of sensor technology, wireless communication technology, and automatic control technology to connect the devices distributed in various places through the network to achieve network automation management of the whole home. Today's advanced technologies such as wireless communication technology and wireless sensor networks have a very broad application prospect as they go to monitor various devices or targets in a self-grouping multihop manner and are also able to process this information. The wireless sensor network is the emerging next-generation sensor network, its research began in the late 1990s, and the most representative discussion is a paper entitled "Sensors towards the Wireless Era" in 1999, followed by the International Conference on Mobile Computing and Networking in the United States, put forward the wireless sensor network is the 21st century The idea that wireless sensor networks are a major development opportunity for the 21st century was presented at the International Conference on Mobile Computing and Networking in the United States. After the introduction of middleware technology into wireless sensor networks, several research institutions then started to study different aspects, most of which are focused on the development of middleware for the characteristics of wireless sensor networks, mainly on how to extend the network lifetime. Therefore, the focus of intelligent sporting event LAN research has shifted from wired networks to wireless sensor networks. The scale of the network, the power consumption problem of wireless nodes, the transmission distance between nodes, and the stability of the network will be the key to future research on wireless sensor networks. By integrating wireless sensor network technology and smart sporting events to form the most popular smart sporting event monitoring system, the paper introduces GPRS and CC1110 wireless access technology, studies a wireless sensor network-based smart sporting event remote monitoring system design scheme, and discusses the application of key technologies [2].

The literature discusses the structure of commercial sporting events and the assets owned by each type of entity, based on the concept of the input-output model. There are generally two types of production sectors that combine funding and operation in one exclusive production sector and a production sector that is made by the cooperation of multiple operating entities of the funding and operating parties. The product resources of sports events are the spectator services of human movement combinations created in the production process of sports events and the derivative resources that can be used for reproduction. The event management system is a sports event organization management system for schools, stadiums, enterprises, and institutions, integrating a number of functions such as sports event registration, scheduling, checking and recording, results processing, and report summary printing. The system is flexible, easy to operate, and functional, which can help to deal with the tedious information management work during the games, reduce the workload of the event organizers, and improve the efficiency and quality of work. The main consumers of sports events are the spectators of sports competition performances and the production sectors that use various types of derivative resources of sports events as their reproduction inputs. The definition of sports events based on industrial economics, that is, the organizing committee of the event as the producer, the athletes, coaches, referees, and other labor and all kinds of sports equipment and other capital as inputs, the production of all kinds of human movement for people to watch the action combination of products and their derivatives of an organized, purposeful production activities. The organizers of commercial sporting events are business entities, the mode of operation is marketoriented, their purpose is to make profits, and the transaction objects have a diversity of transaction methods. The literature argues that sporting events are special events that provide competition products and related service products, and their scale and form are governed by competition rules, traditional customs, and a variety of factors.

\section{Related Work}

With the rapid transformation of A country's economic structure, the development space of the sports industry has gradually increased, but due to the relatively lagging development of the sports industry over the years, the problems are still very prominent, such as the mismatch between supply and demand, imbalance in structural layout, and backward supply mode within the sports industry. Since the introduction of the supply-side reform in 2015, many scholars have also continued to study the supply-side reform of the sports industry in depth. The literature suggests [3] that the current development scale of the sports industry in country A is small and the structure is not excellent, and it needs to develop the sports industry vigorously with the opportunity of supply-side reform in country A. The focus of the supply-side structural reform of the sports industry is to make up for the shortcomings to increase the effective supply of sports services and sports products. The literature believes that the starting point of the supply-side reform of the sports industry is that the current supply of sports products and services is insufficient and inefficient to meet the growing and diversified sports consumption needs of the public, and the sports industry has been given the mission of the times to promote economic transformation and upgrading, which has been the development reality of the supply-side reform of the sports industry, and the new concept of development of innovation, coordination, green, open, and sharing should be taken as the guide for action, to promote the supply-side reform of sports industry. According to the literature, there are problems in the development of the sports industry, such as roughness, lowquality level, and insufficient personalized supply. With the theory of supply-side reform as a guide, we should continue to increase the investment in the sports industry, expand the scale and level of the sports industry, focus on optimizing the supply structure of the sports industry, and promote the upgrading [4] and development of China's sports industry. 
The literature believes that China's economy is currently in the key node of structural reform, and the "supply-side reform" pursues an economic structure that matches effective supply and effective demand, and at the same time, it also puts forward new requirements for the development of the sports industry, to grasp the new opportunities brought by the supply-side reform to the sports industry, and the purpose of reducing ineffective supply is only to ensure. The prerequisite for the healthy and sustainable development of sports industry is to increase the "gold content" of the sports industry, and the most fundamental means to achieve the milestone development of China's sports industry is to increase the effective supply [1].

Through combing, it is found that relevant studies on "supply-side reform" in the sports industry have been elaborated, and it is generally believed that the sports industry in China A has ushered in a golden period of development, and the supply-side reform should be used to improve the scale and optimize the structure of the industry, to facilitate the optimal allocation of the factors and resources of the sports industry and let more public enjoy the fruits of development. However, most of the studies are based on the discussion and research of the whole sports industry [5], and there are fewer descriptions of the supply-side reform of mass sports events. The literature studied the theoretical framework and operation model of sports event operation, analyzed the current situation of sports event operation in country A concerning advanced sports event operation and management concepts, and proposed to build a sports event operation and management model with characteristics for optimization of sports event management system. The literature studied the market competitiveness of sport competition products in country A based on global research results about market competitiveness [6]. The problem was studied, the factors affecting the market competitiveness of sports competition products and force were analyzed, the evaluation system of the market competitiveness of sports competition products was pointed out, and an empirical study was done [7]. The literature studied the market competitiveness of sports competition products in three cities from three dimensions: competitive sports, mass sports, and sports industry, and designed the corresponding evaluation index system, and the analysis gave an effective way to improve the market competitiveness of urban sports [8].

In the planning of sporting event management, more research is done on how to plan the sporting event reasonably, inside the sporting event can also only rely on reasonable planning guidance screens to provide available information, therefore, cannot directly access the information of available management bits, need to stay on the main road inside the sporting event for a longer time to cruise around to find available management provided sporting event solutions, mostly through the entrance of the sporting event. Some researchers in country A have also studied the model of car parking space selection and accurate guidance methods inside sporting events. For example, the literature based on the Dijkstra algorithm to achieve not only accurate parking guidance in sports events, but also guide the parkers to a certain management channel, can not determine the specific management position [9], and the parkers need to find the available management position after entering the channel, in the longer channel, still need to spend more time cost of the parkers, and at the same time in the narrow channel stay too long, will also increase the possibility of the channel the possibility of congestion. The current wireless sensor network-based intelligent guidance system within sporting events is still lacking in terms of low-power design, data transmission reliability, and accurate guidance for event broadcast. The nodes are battery powered, and the deficiencies in low-power design will reduce the service life of the nodes and increase the maintenance cost of the system, while the reliability of data transmission precise guidance methods are directly related to the quality of guidance services provided by the parking guidance system and determine the operational efficiency of the sports event broadcast and the degree of humanized experience for the viewers [10].

\section{Optimization of Sports Event Management System Based on Wireless Sensor Network}

3.1. Specific Optimization of Wireless Sensor Networks. Different interpretations of the definition of sporting events have been proposed in different literature based on different perspectives. There are opportunities and challenges in the organization of large sporting events, and the local city of A needs to introduce new ideas and new technologies in the one-stop service of large sporting events. Wireless sensor network-based technology is an emerging technology, and although there are differences in the areas of application, the concept of digitalization and information technology fits with the one-stop service for large sporting events. It is important to explore the correlation between the two in-depth studies and to clarify the significance of their integration [11].

In this paper, the overall framework design of the intelligent guidance system for events is carried out according to the problems unearthed in the analysis of the intelligent guidance requirements for sports event management, and the overall framework of the system is shown in Figure 1. This intelligent guidance system is mainly divided into two parts in the overall framework, which are the event scheduling system and the event broadcast system [12]. The wireless sensing subsystem is mainly responsible for collecting the status information of the event broadcast using wireless sensing network technology. The platform subsystem uses a server to process the data of the state information collected by the wireless sensing subsystem to achieve the optimal way of sports event management to maximize efficiency [13].

To facilitate understanding, a video from the structure can be divided into scenes, shots, and video, as shown in the figure; the scene consists of some related shots, shots are used to record a specific time and place within the continuous behavior and consists of spatial and temporal continuous image towel chaste, and so a video clip is a series of the continuous image in the chaste. The sports event concept is the most basic unit in the video structure, the image towel chaste according to a certain rate of play can form the frequency observed by the human eye, the information 


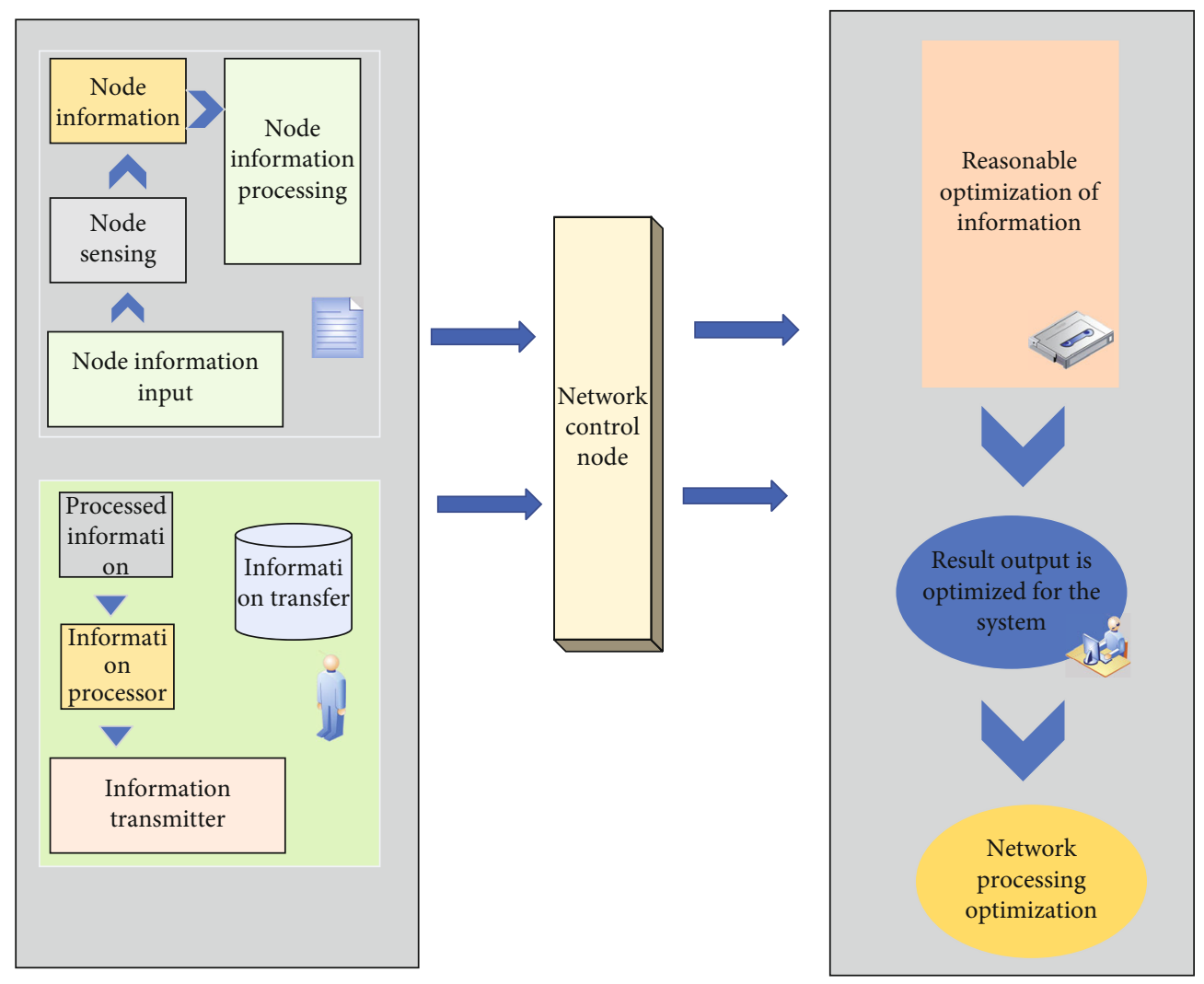

FIGURE 1: Framework diagram of the optimization system.

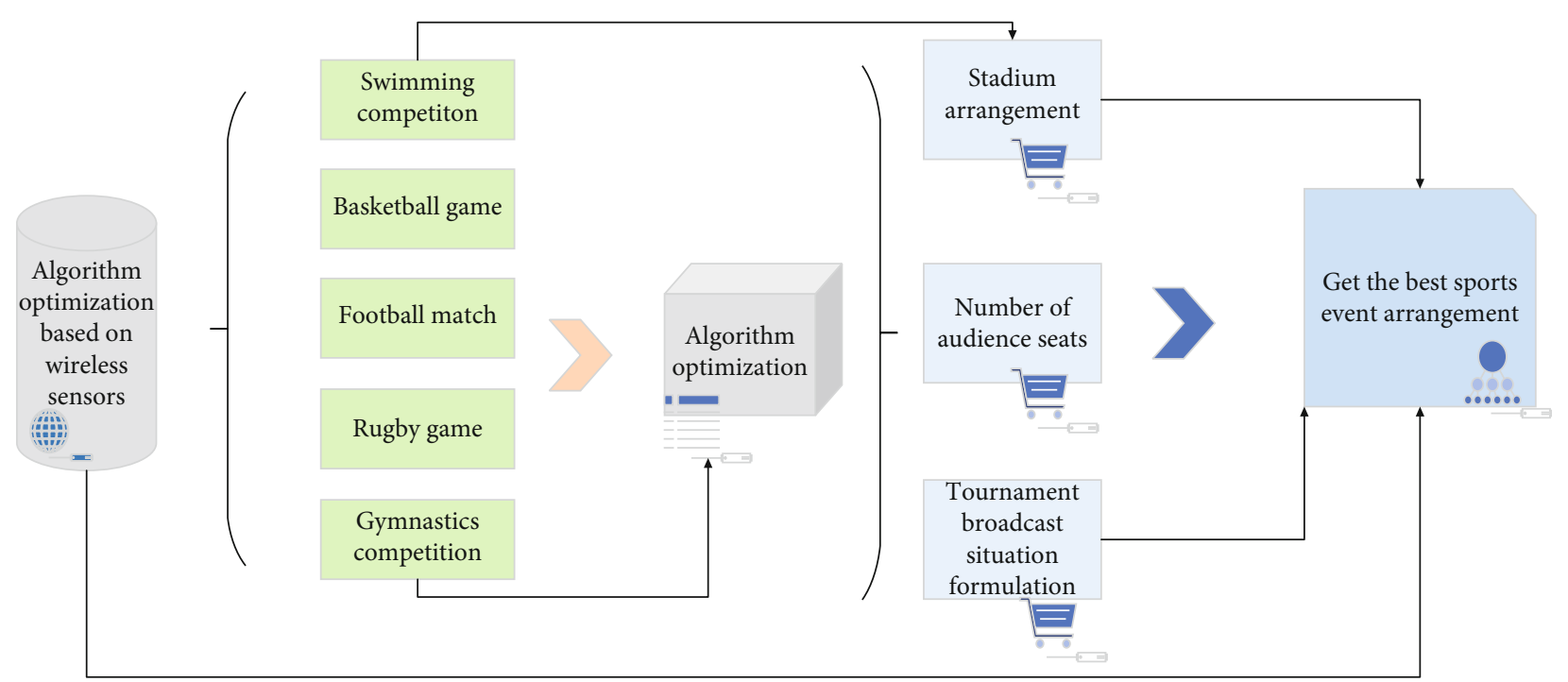

Figure 2: Process flow diagram for optimization of sports event management.

abstraction of the image response to the entire video content, automatic analysis, and processing of video data, generally built on the image dump.

On the technical means based on wireless sensor networks for sporting event management are discontinuous points or points with drastic changes in the gray value of the image, reflected by the gradient of the gray distribu- tion of the image pixels. The edge is a fundamental feature about the boundary, carrying a large amount of information and occupies an important role in the field of image processing, analysis, and recognition. Edge features are easy to extract, computationally small, and fast, so edge detection has always been one of the research hotspots in computer vision. Edge extraction methods are generally 


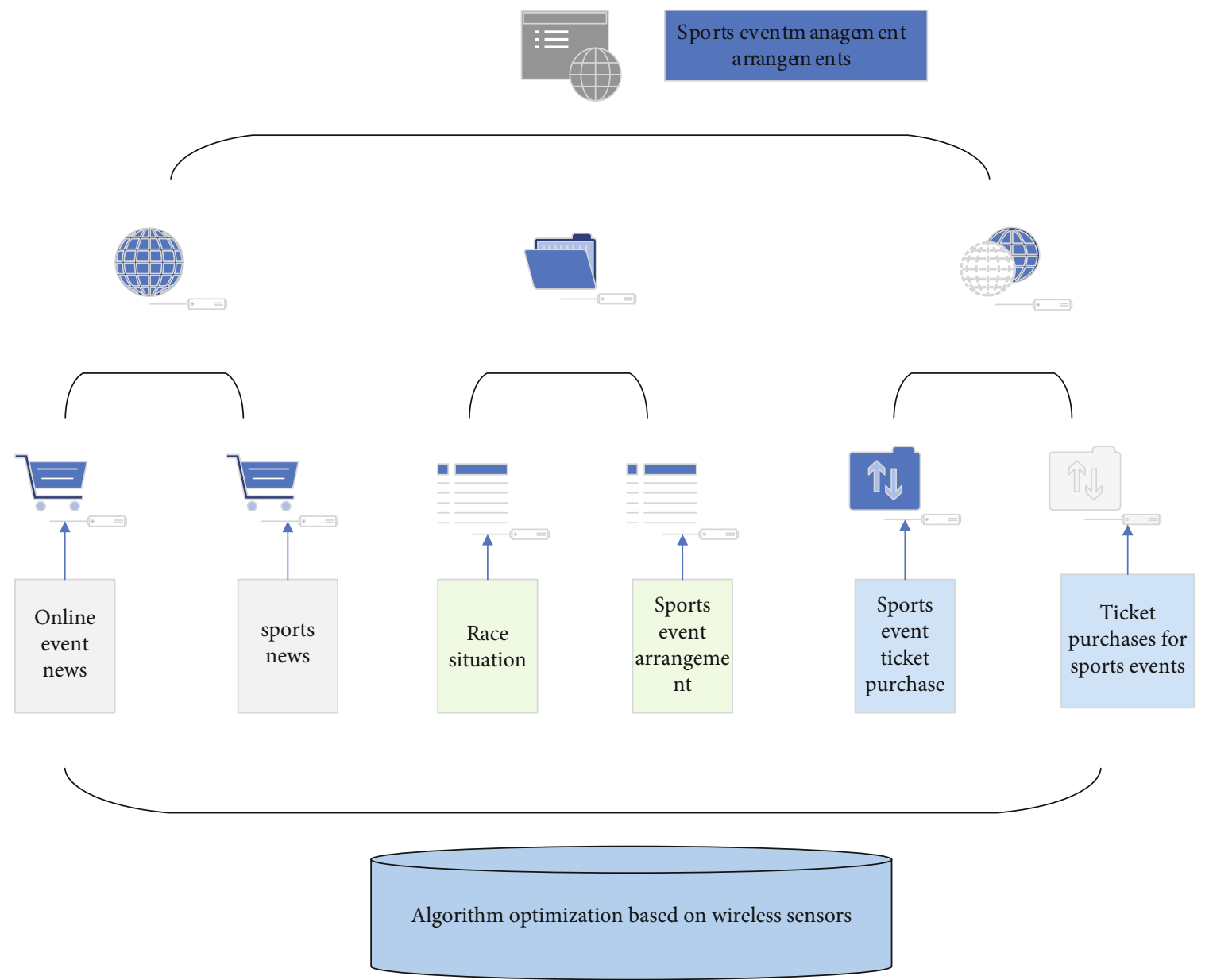

Figure 3: Sports event management topology.

based on individual pixels of an image, and the edges are detected by calculating the variation between gray levels in the local neighborhood of the image using first-order or second-order directional derivative. Traditional edge detection algorithms include operators, operators, subs, and operators, through this type of edge operators to extract the edge of the image has the characteristics of simple operation, easy to implement, etc., but due to the use of differential calculation, often more sensitive to noise, vulnerable to interference, the extracted edge is not fine enough, in the practical application will be subject to certain limitations. The principle is shown in Figure 2.

In contrast, a new edge detection method is proposed with excellent performance; the edge detection operator uses a double threshold detection and connected edge based on gradient values, which can suppress noise and has good results, and is also a very popular edge operator nowadays, solving the three standard problems of signalto-noise ratio criterion, localization accuracy quasiwarping, and unit response criterion; and using optimization theory to obtain the optimal edge template for detection, the whole edge detector algorithm can be divided into four steps as follows [14]:
(1) A Gaussian filter is used to convolve with the original image to eliminate noise and smooth the image with the following Gaussian function. Gaussian filtering is a linear smoothing filter that removes Gaussian noise, the effect of which is to reduce the sharp changes in the gray scale of the image, that is, the image is blurred. Gaussian filtering works very well for suppressing noise that obeys a normal distribution, at the cost of making the image blurry. Of course, sometimes the purpose of smoothing and filtering an image is to make it blurred

$$
F(\mathrm{x}, \mathrm{y})=\lim _{n \longrightarrow \infty} \sum_{i=1}^{n} X_{i} Y_{i}+e^{-(x-\mu) / \sigma}
$$

(2) The first-order differential operator is used to calculate the pixel points in the image with the following equation: 


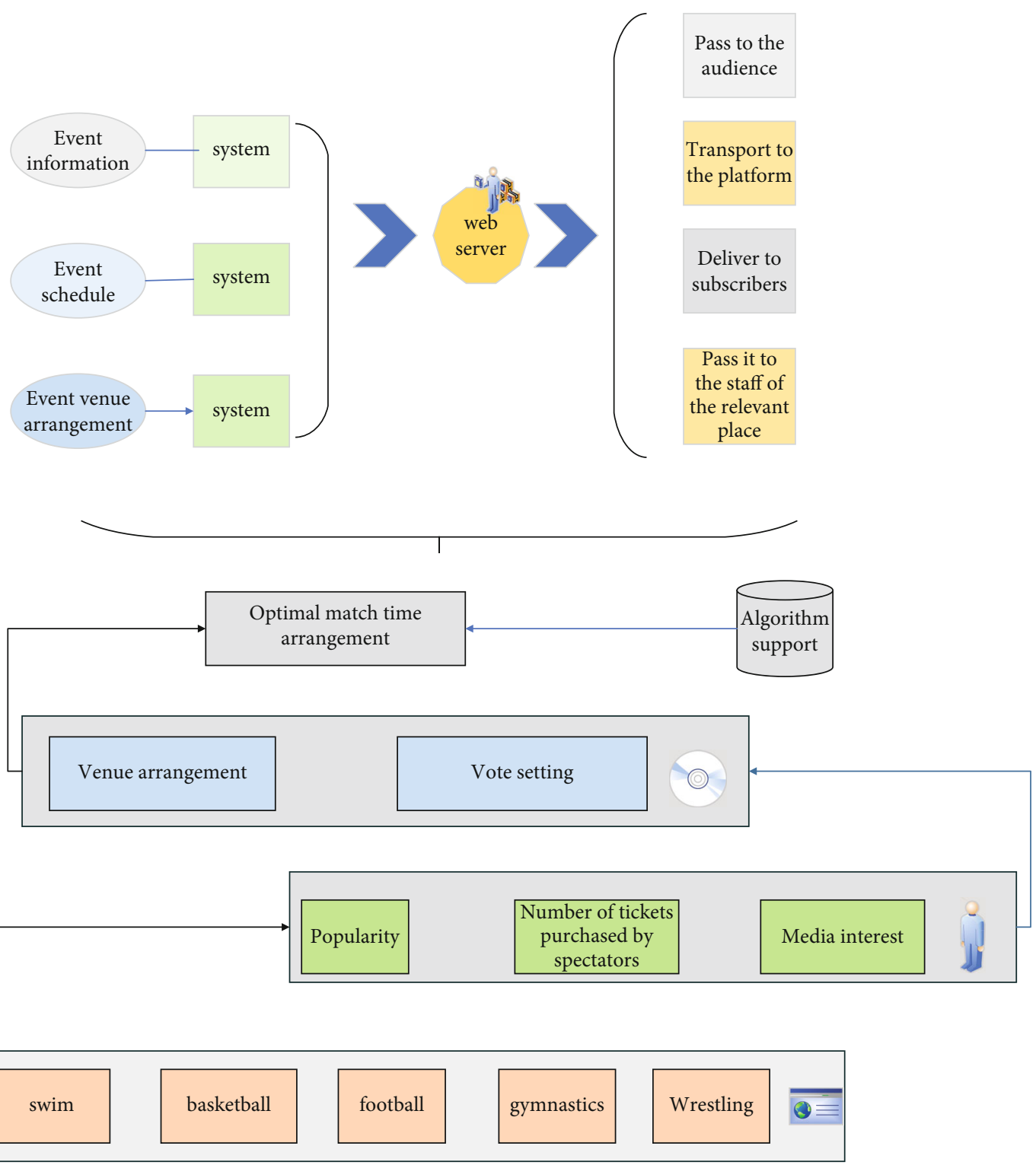

FIgURE 4: Overall data flow of information.

$G(\mathrm{x}, \mathrm{y})=\frac{1}{2} \lim _{n \longrightarrow \infty} \sum_{i=1}^{n} X_{i} Y_{i}+\lim _{n \longrightarrow \infty} \sum_{i=1}^{n}\left(X_{i}-\bar{X}\right)^{2}$

(3) Nonmaximum suppression of gradient amplitude: the above process only gets the global gradient is not enough to determine the edge; this step that is to refine the image of the gradient amplitude of the extreme value region, retain the local gradient amplitude of the largest point, to suppress the nonmaximum value, to achieve the effect of refining the edge. First, based on the neighborhood of $G$ as the center of the direction, the neighborhood is interpolated in the $G$ gradient direction [15], and then, the center point $G$ is compared with the interpolation size of the two adjacent gradient magnitudes in its gradient direction, and if the magnitude of the center point of the neighborhood is greater than or equal to the interpolation result in its gradient direction, it can be regarded as the initial edge point, and if it is less than this value, it is regarded as a nonedge point, and its value is regarded as zero, and after passing this step, the image can be obtained. And a suitable method for sports event optimization can be obtained. First-order differential operators are generally done by means of volume sets with the help of null field differential operators, but in practice, derivatives are found in digital images using difference approximation differentiation. The gradient corresponds to the first-order derivative and the gradient operator is a first-order derivative operator: 


$$
A=\arccos \theta \cdot \lim _{\Delta x \rightarrow 0} \frac{\Delta y}{\Delta x}=\arccos \theta \cdot y_{x}
$$

(4) To overcome the effect of noise and improve the accuracy, the edge idea uses the algorithm of double thresholding to detect and connect the final edges, setting two thresholds and, and by the thresholds can be split into two threshold edge images. Its formula is

$$
M=\cos \theta \lim _{\Delta x \longrightarrow 0} \frac{\Delta y}{\Delta x} \cdot \frac{\delta y}{\delta x} \cdot \frac{\partial^{2} \Omega}{\partial u \partial v}=y_{x} \cos \theta \cdot \frac{\delta y}{\delta x} \cdot \frac{\partial^{2} \Omega}{\partial u \partial v}
$$

\subsection{Optimization Measures for Sports Event Management} Systems. In the process of managing sports events based on a unified resource relationship model, it is proposed that a large sports event management system needs to have information management functions such as basic information, personnel, events, venues, game schedules, tournament systems, and constraints and has the functions of executing game scheduling and schedule execution. According to the functional correlation between the subordinate systems, the large-scale sports event management system is divided into three parts: match information support, match decision schedule [16], and match site execution. The competition information support module provides supporting information for the operation of the large sporting event management system, which contains athlete information, event information, venue facilities, and other personnel information. The competition decision scheduling module is the orchestration station of the major sports event management system, covering the master plan of the event, the regulation of the competition, and the scheduling on-site. The competition field execution module is the core part of the operation of a major sporting event, which can enter results in realtime, generate athlete rankings, specify the groups, start times, venues, and participants, as well as automatically achieve advancement to the next round according to the results, and allow for the publication of results. In this system, the exact correspondence between the envisaged events and the results is achieved and the smooth running of the event can be accomplished. Optimization problems are divided into two categories: functional optimization problems and combinatorial optimization problems, where the object of functional optimization is a continuous variable in a certain interval, and the object of combinatorial optimization is a discrete state in the solution space. Typical combinatorial optimization problems include traveler's problem, processing scheduling problem, backpacking problem, boxing problem, graph coloring problem, and clustering problem.

At present, country A is in an era where mobile Internet and traditional Internet coexist, and the use of mobile Internet is increasing, and there will be Internet in various industries and fields to a greater or lesser extent. Popular sports events focus more on improving the physical quality of the

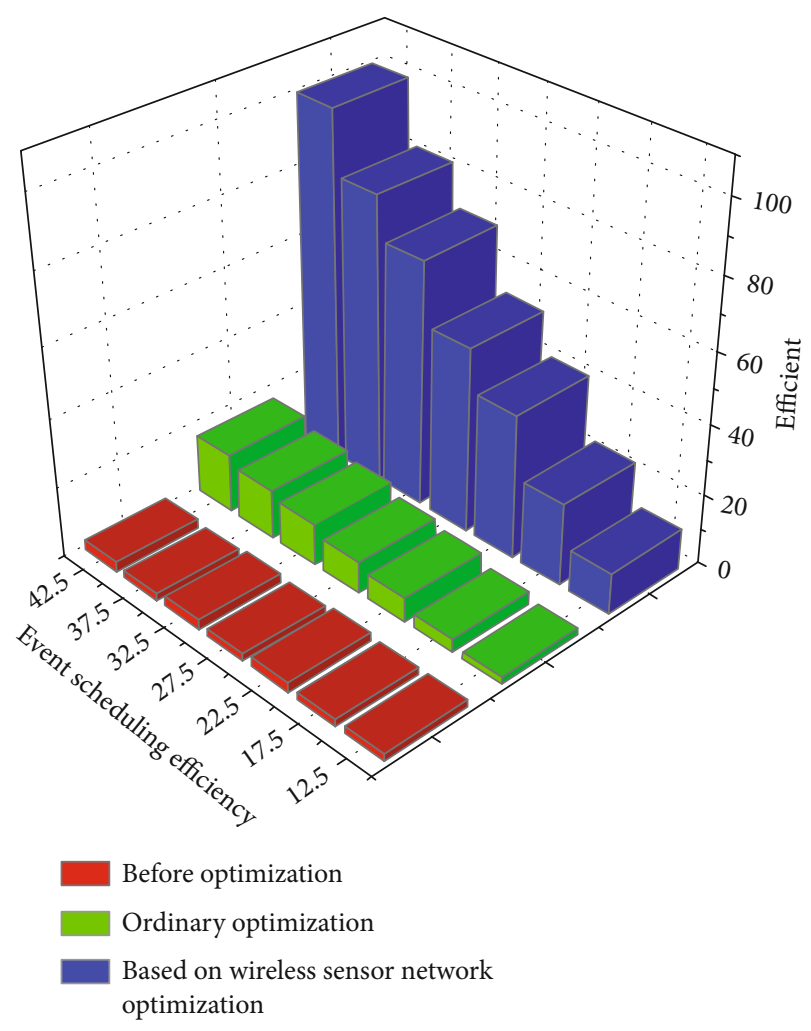

Figure 5: Optimize the comparison chart.

masses, developing sports, expanding the number of amateur athletes, and improving the benefits of the event in terms of economy, operation, publicity, and the branding effect of the city, event, or athlete. In the context of the Internet era, more and more research has been conducted on the electronic system of information management of sports events, and the information management of sports events has gradually developed in the direction of networking, electronic, and intelligence. Through the research and analysis of international tennis events, the literature concludes that it has the characteristics of obvious structural stratification, close internal interconnection, high degree of external openness, and structural consolidation and stability and then analyzes the problems of low internal structural correlation, inconspicuous structural hierarchy, and low degree of structural openness in the structure of sports events and puts forward corresponding suggestions combined with the revelation of the structure of international tennis events. It is shown that badminton and tennis have a correlation in the event structure, and summarizing the event structure of tennis can bring new thinking and new direction for the optimization of the event structure of badminton in China. With the increasing concern for sports health and the rapid growth of the number of various sports and fitness venues, sports venues urgently need to develop an intelligent sports management system for the convenience of user exercise and communication, which includes sports venue recommendation and booking, sports course registration, intelligent sports program development, and recommendation, sports experience sharing, nearby people communication and ball 
TABLE 1: Sports event information table.

\begin{tabular}{lccc}
\hline Content & Venue arrangement & Before optimization & Optimized \\
\hline 1 & Number of audience seats & 90 & 99 \\
2 & Event broadcast arranged by the media & 89 & 92 \\
3 & Final effect & 88 & 97 \\
4 & Audience satisfaction & 82 & 95 \\
5 & Competition presentation & 70 & 99 \\
6 & Event broadcast rate & 89 & 99 \\
\hline
\end{tabular}

appointment, tournament management, booking management, order management [17], athlete management, personal management, and other functions, and to ensure that the information in the use of the system can be accurately and smoothly transmitted to facilitate users to make venue reservations and exercise. The network topology diagram of the intelligent sports management system is shown in Figure 3.

Based on the organizational structure of the public service body for major sporting events, the public service organization for major sporting events is divided into three levels: the first level is the organizing department for major sporting events, the second level is the director, who is responsible for coordinating the work of all departments, and the third level is the functional departments. The Expert Advisory Department and the Inspectorate Department are relatively independent departments that are not involved in the preparation of specific events but only provide professional advice for decisions related to the public service organization. The composition and distribution of functions are different for different levels and scales of sporting events. The full range of services and support system for large sporting events contains many aspects, based on the public sports, event viewing [18], and participation aspects, the number of people participating in watching sporting events is gradually increasing, to cope with the influx of tourists in the short term, the event host region must establish a high-quality logistical support system. Figure 4 shows the overall data flow of information.

The overall data flow of the system is represented by the total data flow diagram, but if you want to describe some internal processing and details of the system, then you can use the branch data flow diagram. By using the system branch data flow diagram to describe the interaction process between user APP, PC management system, and Web server, the user accesses the Web server through APP to make reservation and other operations conveniently and quickly, at this time, the PC support system also accesses the latest data of the server Firstly, in terms of reception, the cooperation with travel agencies can be adopted, so that the tourists can also visit other scenic spots in the hosting area after watching the game. The second is the security and medical security of the event [19], the organizer should take the initiative to get in touch with the local public security bureau and health and medical departments, to get more professional and official logistical support and guidance for the event, to ensure the safety and medical health of all the

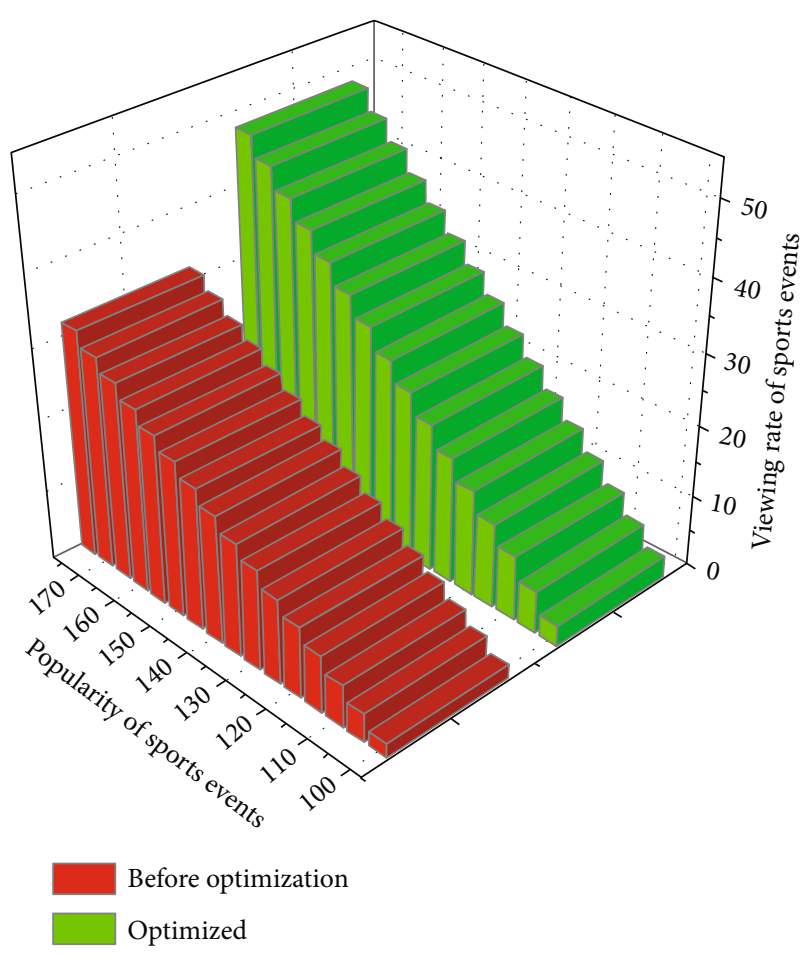

Figure 6: Optimized efficiency graph after optimization.

people involved in the race; again is the maintenance of the venue, stage design and event performances, etc., to ensure the professionalism and safety of the field. Finally, a professional and stable team of volunteers should be formed, and through systematic training, the volunteer service team should provide comprehensive and three-dimensional service and logistical support for the event. Even if we are well prepared, there will still be more or less unexpected events, big or small, in the preparation or event holding stage, so we should do a good job of an emergency handling plan [20]. Optimization methods are a mathematical approach, which is a general term for a number of disciplines that study how to seek certain factors to optimize a given metric under a given constraint. The essence of most machine learning algorithms is to build optimization models and optimize the objective function by optimization methods to train the best model. Common optimization methods include gradient descent, Newton's method and proposed Newton's method, and conjugate gradient method. 


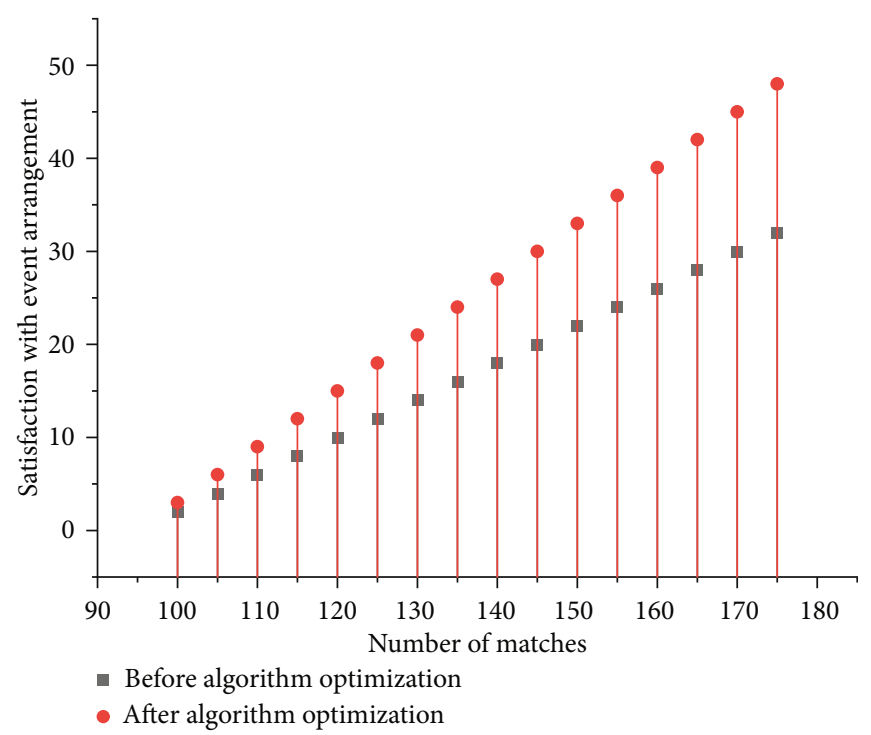

FIGURE 7: Efficiency graph for continued optimization after algorithm improvement.

\section{Experimental Results and Analysis}

4.1. Experimental Results. The architecture of an intelligent sports management system is a closed-loop structure, which contains five parts: user APP, venue APP, athlete APP, WeChat public number, and PC management system. The user APP is for sports fans, that is, the guests and service users of sports venues, through which users can easily and quickly perform some operations and enjoy services, mainly online booking, and mobile payment. With the development of the network from PC to mobile, especially the leaders and directors of the venues, they do not always sit by the computers in the venues all day, so through this APP, they can do office work and check the revenue statistics and various operation analysis of the venues anytime and anywhere and master the dynamics of the venues 24 hours a day. Athletes APP is mainly for athletes who come to the gym for sports and exercise. Through this APP, athletes can see the training plan and some data customized by the coach, such as historical performance analysis and height and weight temperature records. The function of the WeChat public number is similar to that of user APP, and it is mainly used by users, but it is more convenient and fast, especially for some people who do not often go to sports venues, they do not even need to download and install APP, they just need to pay attention to the corresponding WeChat public number to realize the functions of venue booking and bill payment, etc. The PC management system is one of the important components of an intelligent sports management system, it is mainly used by the staff of the venue, and its functions include all the functions of the APP of the venue but not only that, such as the front desk registration when users come to exercise and the checkout and service for some casual customers who do not use the intelligent management system. Figure 5 optimizes the comparison chart.

The role of the database of this system is divided into two main parts; one is for recording and storing venues, courses, users, booking. It is easy to retrieve and access the various information on orders, operations, etc., and the connections and relationships between them, as well as the information on PC and mobile. The second is to use some advanced technology such as big data, data mining techniques to explore the value of data The current technology related to big data is very hot, and the use of big data technology in China started late, some places are not perfect, many domestic companies are moving in this direction, and the use and development of big data can not be separated from the collection and storage of data. The database tables in the intelligent sports management system include venue information table, course information table, user information table, venue basic information table, sports information table, coach information table, staff information table, and operation data table. The following is a brief list of some database table structures. The venue information table is mainly used to describe the information and attributes of the venue, including venue name, address, description, price, discount, region, evaluation, opening hours, and environment, as shown in Table 1.

By analyzing the problem of venues failing to provide professional guidance and recommended exercise programs to users, the sports and health sector was selected. Research and modeling were conducted to construct an ontology knowledge base. Then, some information about the user such as age, height, weight, and health status, using a combination of case-based and rule-based reasoning to generate exercise programs and recommend them to users. Exercise the program recommendation function is not only a practical demand of users for intelligent movement but also improves the information and professionalism of the venue and increased user stickiness. Its data graph obtained through the optimization of the wireless sensor networkbased sports event system is shown in Figure 6.

Experiment first with the original Ullmann algorithm, i.e., using ALL-DEG as the reference criterion, to see if 


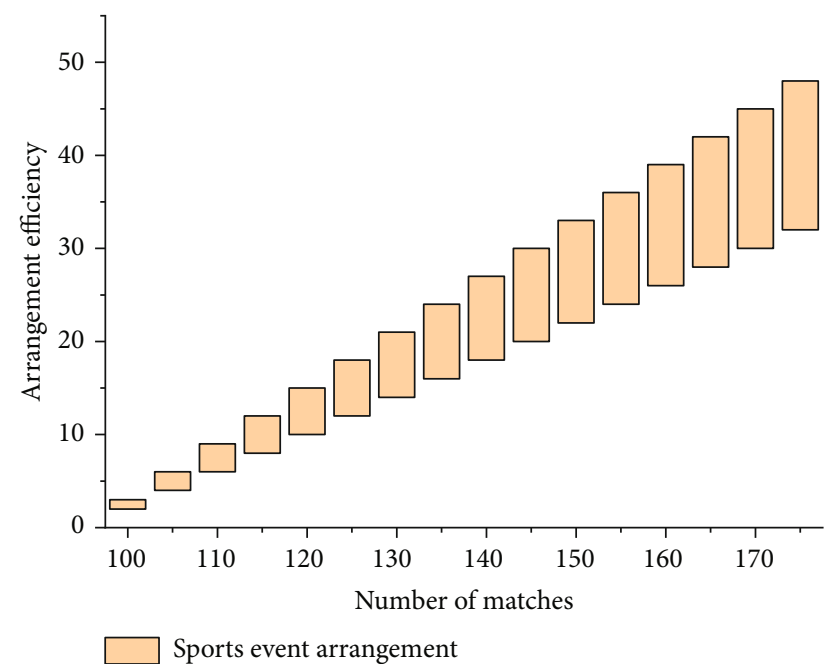

Figure 8: Performance comparison before and after algorithm improvement for the dataset scenario.

the new order improves overall performance. The scenario *-DEG denotes the selection process of different candidate sets $C$ with DEG as the selection criterion for a single candidate vertex; the scenario ALL-* denotes the selection process of the candidate set $C$ with ALL as the selection criterion for a different single candidate set. Among the ranking methods shown in Figure 6, ALL-DEG is the most effective. The main reason for this is that the refinement process of the Ullmann algorithm is fully refined, and it works in the global compatibility matrix.

4.2. Experimental Analysis. In contrast, the ALL-DEG sorting is not as efficient as the other methods when it comes to local refinement. Figure 7 shows that in the $*$-DEG scenario, the NEIGH and RDEG methods exhibit better performance than the DEPTH and ALL methods. Similarly, in the ALL-* method, CNC and ONC perform better than DEG and ECC. Figure 7 shows that in the NEIGH-* and RDEG-* criteria, CNC performs the best, followed closely by DEG. Based on these results, we chose RDEG-CNC as the final sorting method of the algorithm.

Optimization of sports event management system based on wireless sensor network, the running time of the matching process before and after the improvement of Ullmann algorithm for different size query graphs; in this scenario, Figure 8 indicates that the improved Ullmann algorithm is still better than the Ullmann algorithm in terms of performance, overall. But the overall result is not as good as in the US Patents dataset scenario, which also shows that Spark is more suitable for iterative operations on large datasets and can be a good study for tournament optimization.

\section{Conclusion}

With the development of sensor technology, microelectronics technology, low-power wireless communication technology, and other modern information technology, wireless sensor network as a new application model of wireless network has been widely studied. Due to the unique advantages of wireless sensor networks such as low cost, low power consumption, self-organizing network, fast, and flexible, they have been widely used in military, industrial and agricultural, environmental, and other fields. With the development of sensor technology, microelectronics technology, low-power wireless communication technology, and other modern information technology, wireless sensor network as a new application model of wireless network has been widely studied. Due to the unique advantages of wireless sensor networks such as low cost, low power consumption, selforganizing network, fast, and flexible, they have been widely used in military, industrial and agricultural, environmental, and other fields. For the limited energy characteristics of wireless sensor networks, data fusion techniques are introduced in the network to fuse the data collected by sensor nodes. This paper provides a summary overview of wireless sensor networks and data fusion techniques based on improved routing protocols, focusing on their characteristics, classification, and application roles.

\section{Data Availability}

The data used to support the findings of this study are available from the corresponding author upon request.

\section{Conflicts of Interest}

The authors declare that they have no known competing financial interests or personal relationships that could have appeared to influence the work reported in this paper.

\section{Acknowledgments}

This work was supported by Basic research fund for excellent teachers of the Ministry of education of the People's Republic of China: Research on the construction and practice of university sports informatization under the background of big data and Internet plus Era (No. 2-9-2018-336).

\section{References}

[1] G. Babu Loganathan and S. I. Hadi, "EERP: intelligent cluster based energy enhanced routing protocol design over wireless sensor network environment," International Journal of Modern Agriculture, vol. 10, no. 2, pp. 1725-1736, 2021.

[2] S. Bhushan, M. Kumar, P. Kumar, T. Stephan, A. Shankar, and P. Liu, "FAJIT: a fuzzy-based data aggregation technique for energy efficiency in wireless sensor network," Complex \& Intelligent Systems, vol. 7, no. 2, pp. 997-1007, 2021.

[3] S. Manikandan and M. Chinnadurai, "Effective energy adaptive and consumption in wireless sensor network using distributed source coding and sampling techniques," Wireless Personal Communications, vol. 118, no. 2, pp. 1393-1404, 2021.

[4] R. P. Ojha, P. K. Srivastava, G. Sanyal, and N. Gupta, "Improved model for the stability analysis of wireless sensor network against malware attacks," Wireless Personal Communications, vol. 116, no. 3, pp. 2525-2548, 2021. 
[5] S. Pragadeswaran, S. Madhumitha, and S. Gopinath, "Certain investigations on military applications of wireless sensor networks," International Journal of Advanced Research in Science, Communication and Technology, vol. 3, no. 1, pp. 14-19, 2021.

[6] P. Rawat, S. Chauhan, and R. Priyadarshi, "A novel heterogeneous clustering protocol for lifetime maximization of wireless sensor network," Wireless Personal Communications, vol. 117, no. 2, pp. 825-841, 2021.

[7] D. D. S. David, R. Parthiban, D. Jayakumar et al., "Medical wireless sensor network coverage and clinical application of MRI liver disease diagnosis," European Journal of Molecular \& Clinical Medicine, vol. 7, no. 9, pp. 2559-2571, 2021.

[8] J. Li, M. Gao, J. S. Pan, and S. C. Chu, "A parallel compact cat swarm optimization and its application in DV-hop node localization for wireless sensor network," Wireless Networks, vol. 27, no. 3, pp. 2081-2101, 2021.

[9] S. Nagaraju, L. J. Gudino, N. Tripathi, V. Sreejith, and C. K. Ramesha, "Mobility assisted localization for mission critical wireless sensor $\mathrm{n} \backslash$ etwork applications using hybrid area exploration approach," Journal of King Saud University-Computer and Information Sciences, vol. 33, no. 5, pp. 608-618, 2021.

[10] R. Shakila and B. Paramasivan, "An improved range based localization using whale optimization algorithm in underwater wireless sensor network," Journal of Ambient Intelligence and Humanized Computing, vol. 12, no. 6, pp. 6479-6489, 2021.

[11] J. Thyagarajan and S. Kulanthaivelu, "A joint hybrid corona based opportunistic routing design with quasi mobile sink for IoT based wireless sensor network," Journal of Ambient Intelligence and Humanized Computing, vol. 12, no. 1, pp. 991-1009, 2021.

[12] S. S. Mohar, S. Goyal, and R. Kaur, "Optimized sensor nodes deployment in wireless sensor network using bat algorithm," Wireless Personal Communications, vol. 116, no. 4, pp. 28352853, 2021.

[13] C. Singhal and V. Patil, "HCR-WSN: hybrid MIMO cognitive radio system for wireless sensor network," Computer Communications, vol. 169, pp. 11-25, 2021.

[14] Y. Cao and H. Mao, "High-dimensional multi-objective optimization strategy based on directional search in decision space and sports training data simulation," Alexandria Engineering Journal, vol. 61, no. 1, pp. 159-173, 2022.

[15] H. Shingrakhia and H. Patel, "Emperor penguin optimized event recognition and summarization for cricket highlight generation," Multimedia Systems, vol. 26, no. 6, pp. 745-759, 2020.

[16] N. Djajasinga, B. A. Wibowo, and R. R. Prihatmantyo, "Management and engineering optimization of car free day activities in the Simpang Lima Gumul Monument Area in Kediri Regency,” DEGRES, vol. 20, no. 2, pp. 155-166, 2021.

[17] S. D. Kim, J. C. Kim, and J. Bachman, "Optimization of international sport event tournament schedules: a managerial view on the scheduling fairness of the world baseball softball confederation premier 12 tournament," Journal of Physical Education, vol. 7, no. 2, pp. 9-16, 2020.

[18] T. Li, J. Sun, and L. Wang, “An intelligent optimization method of motion management system based on BP neural network," Neural Computing and Applications, vol. 33, no. 2, pp. 707-722, 2021.
[19] X. Ma, H. Cui, and K. Tang, "Supply-side reform measures of public sports service from the perspective of public health promotion," Revista Brasileira de Medicina do Esporte, vol. 27, no. spe, pp. 53-55, 2021.

[20] V. Kanwar and A. Kumar, "DV-hop localization methods for displaced sensor nodes in wireless sensor network using PSO," Wireless Networks, vol. 27, no. 1, pp. 91-102, 2021. 